\section{ORIGINAL RESEARCH}

\author{
N. Gaillard \\ C. Schmidt \\ V. Costalat \\ J.P. Bousquet \\ C. Heroum \\ D. Milhaud
}

A. Bonafe

C. Arquizan

\title{
Hemorrhagic Risk of Recent Silent Cerebral Infarct on Prethrombolysis MR Imaging in Acute Stroke
}

BACKGROUND AND PURPOSE: EIH is a rare complication after thrombolysis in patients with acute stroke, occurring in brain regions without visible ischemic change on pretreatment imaging. $\mathrm{RSCls}$ can be detected by multimodal MR imaging and might be associated with an increased risk of HT postthrombolysis, related to BBBD. We aimed to assess the incidence of $\mathrm{RSCl}$ on pretreatment MR imaging and the subsequent risk of $\mathrm{HT}$ within $\mathrm{RSCl}$ areas on follow-up $\mathrm{CT}$ performed $<36$ hours after rtPA administration and on additional cerebral imaging before patient discharge.

MATERIALS AND METHODS: Pretreatment MR imaging was retrospectively analyzed from consecutive patients with stroke who received intravenous or intra-arterial rtPA for 2 years. $\mathrm{RSCl}$ was defined on MR imaging as a parenchymal area markedly hyperintense on FLAIR, different from the hyperacute infarct, and mildly-to-markedly hyperintense on DWI or enhanced on postgadolinium T1WI imaging.

RESULTS: Eighty-six patients with a median age of 66 years and a median NIHSS score on admission of 15 were studied; $66.3 \%$ received rtPA intravenously. The presence of $\mathrm{RSCl}$ was identified in 10 patients $(11.6 \%)$ and was associated with large-vessel-disease etiology $(40 \%$ versus $5.3 \%, P<.001)$ on univariate analysis. No HT was identified within the $\mathrm{RSCl}$ areas on any follow-up cerebral imaging.

CONCLUSIONS: These preliminary results require validation but suggest that small RSCls are rather frequent and might not pose a higher risk of postthrombolysis HT.

ABBREVIATIONS: BBBD $=$ blood-brain-barrier disruption; ECASS $=$ European Cooperative Acute Stroke Study; EIH = extraischemic hemorrhage; FFE = fast-field echo; $\mathrm{HI}=$ hemorrhagic infarction; $\mathrm{HT}=$ hemorrhagic transformation; IQR = interquartile range; IS = ischemic stroke; $\mathrm{PH}=$ parenchymal hemorrhage; $\mathrm{RSCl}=$ recent silent cerebral infarct; $\mathrm{SE}=$ spin-echo; $\mathrm{SICH}=$ symptomatic intracerebral hemorrhage; TOAST $=$ Trial of Org 10172 in Acute Stroke Treatment

T hrombolysis is currently an approved therapy for acute IS but increases the risk of SICH, which occurs in 5\%-10\% of patients. ${ }^{1-3}$ Single or multiple EIHs, occurring in regions without visible ischemic change on pretreatment $\mathrm{CT}$ and remote from the site of the acute ischemia, account for approximately $20 \%$ of SICHs after intravenous rtPA in patients with stroke. ${ }^{4}$ This represented an absolute risk of $1.3 \%$ in the NINDS ${ }^{5}$ and $2 \%$ in the ECASS II studies, respectively. ${ }^{4}$ Factors leading to EIH after thrombolysis remain largely speculative with 1 potential mechanism being HT of 1 or multiple RSCIs associated with subacute BBBD. A history of stroke in the past 3 months is currently a contraindication for rtPA in Europe and North America, due to the fear of an increased risk of intracerebral hemorrhage related to BBBD. ${ }^{6}$ Multimodal MR imaging can detect $\mathrm{RSCI}^{7}$ and ischemic BBBD with a high sensitivity, ${ }^{8-11}$ but despite its increasing use in acute stroke management, ${ }^{12,13}$

Received November 11, 2010; accepted after revision May 19, 2011.

From the Service de Neurologie (N.G., C.H., D.M., C.A.), Hôpital Gui de Chauliac, Montpellier, France; Service de Neurologie (N.G.), Hôpital Saint Jean, Perpignan, France; Service de Neuroradiologie (C.S., V.C., A.B.), Hôpital Gui de Chauliac, Montpellier, France; Département de Biostatistique, Epidémiologie Clinique (J.P.B.), Santé Publique et Informatique Médical, Hôpital Caremeau, Nîmes, France.

Paper previously presented at: Eighteenth European Stroke Conference, May 26-29, 2009; Stockholm, Sweden.

Please address correspondence to: Nicolas Gaillard; e-mail: nicolas.gaillard@chperpignan.fr

\footnotetext{
EIndicates article with supplemental on-line tables.

Indicates article with supplemental on-line figures.

http://dx.doi.org/10.3174/ajnr.A2768
}

there are no data regarding the HT risk associated with RSCI postthrombolysis in IS. We thus aimed at assessing the incidence of RSCI on pretreatment MR imaging and the subsequent risk of $\mathrm{HT}$ consequent to routine thrombolysis with rtPA in our stroke center.

\section{Materials and Methods}

\section{Clinical Data and Study Design}

We performed a retrospective analysis of prospectively collected data in all patients with acute IS treated with intravenous or intra-arterial rtPA between January 2007 and December 2008 in our academic stroke center. Patients with a pretreatment cranial CT only or pretreatment MR imaging with the absence of 1 of the required sequences were excluded. A total of 93 patients were treated with rtPA during the study period. Of these, 7 (8\%) were excluded because pretreatment imaging was a CT scan $(n=6)$ or an incomplete MR imaging $(n=1)$, thus limiting the study to 86 patients. We systematically recorded and retrieved the following data: age, sex, NIHSS score on admission, blood test results on admission (including platelet count $<100 \times$ $10^{3} / \mathrm{mm}^{3}$, blood glucose level of $>200 \mathrm{mg} / \mathrm{dL}$, partial thromboplastin time, prothrombin time, and blood creatinine level), symptom-toneedle time, blood pressure at presentation (systolic and diastolic), and intravenous or intra-arterial rtPA administration and a history of high blood pressure, smoking, diabetes mellitus, left cardiac dysfunction, coronaropathy, atrial fibrillation, previous stroke, and thrombophilia status. We also assessed the history of minor IS or TIA in the 3 months preceding the acute IS. 


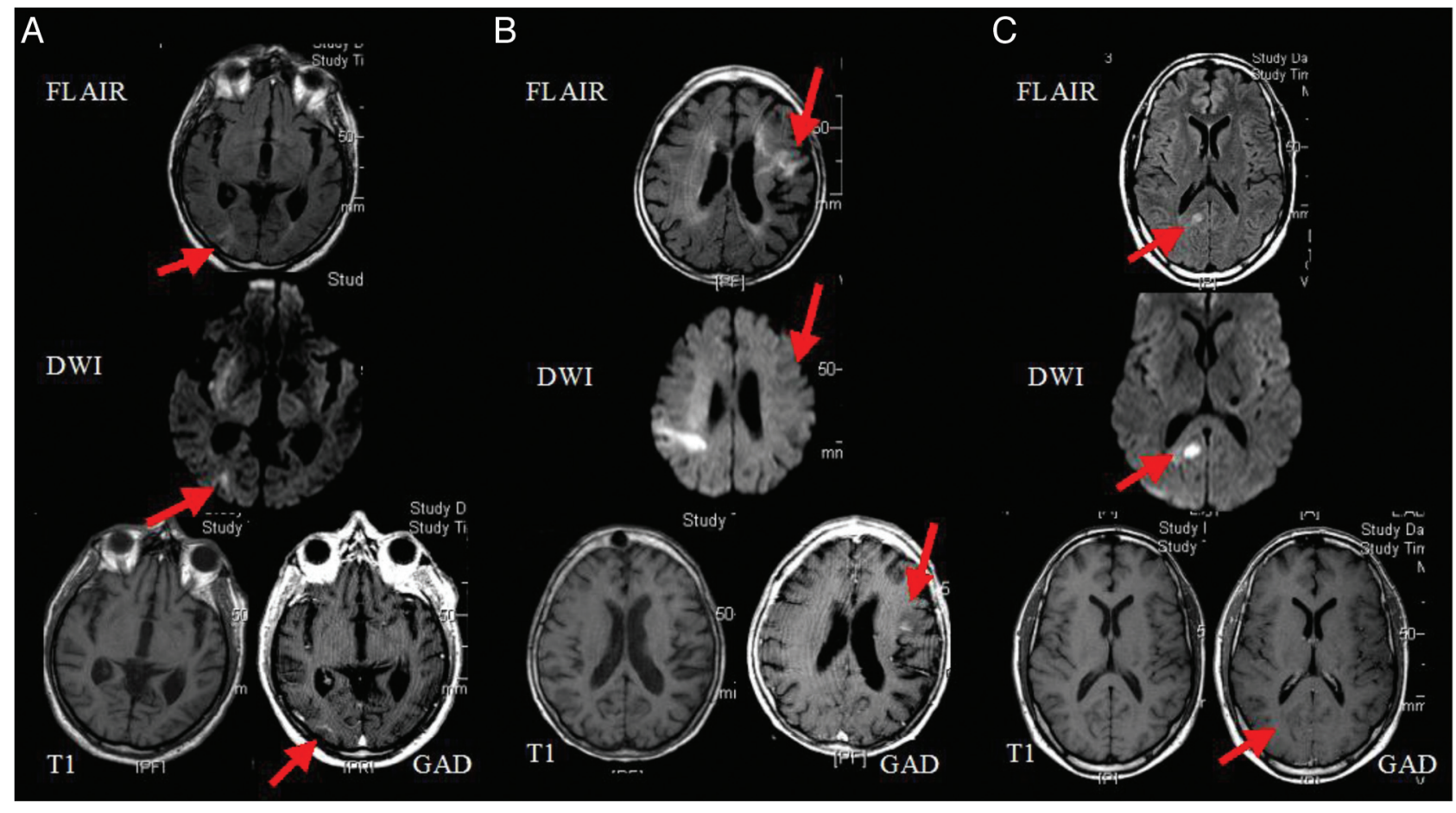

Fig 1. Three different patterns of RSCls defined on MR imaging. $A$, "Gad+/DWI+" indicates area enhanced on postgadolinium T1WI imaging and in hypersignal intensity on DWI. $B$, "Gad +/DWI -" is the area enhanced on postgadolinium T1WI imaging but not in hypersignal on DWI. C, "Gad-/DWI+" is the area unenhanced on postgadolinium T1WI but in hypersignal intensity on DWI. GAD indicates gadolinium.

\section{Thrombolysis Procedure}

Patients with IS were considered for thrombolysis according to local protocol derived from current guidelines and recent evidence-based data. Intravenous thrombolysis with rtPA (full dose, $0.9 \mathrm{mg} / \mathrm{kg}$ ) was considered for patients within 4.5 hours of symptom onset; intraarterial thrombolysis with rtPA (dose ranging from 20 to $50 \mathrm{mg}$ ), whether associated with intra-arterial mechanical thrombectomy or not, was considered between 4.5 and 6 hours postonset or when intravenous rtPA was contraindicated for anterior circulation stroke with large-vessel occlusion and within 24 hours of symptom onset in patients with posterior circulation stroke with basilar artery occlusion. A CT scan was performed 1 day after thrombolysis ( $<36$ hours after rtPA administration) in all patients, with $\geq 1$ further CT or MR imaging, including $\mathrm{T} 2{ }^{\star}$-weighted imaging, performed during the hospital stay when required to assess HT.

\section{Imaging Protocol and Interpretation}

During the study period, multimodal MR imaging was performed in all patients with acute stroke unless contraindicated. MR imaging was the routine imaging technique and was performed on an Intera 1.5T machine (Philips Healthcare, Best, the Netherlands) and included DWI (SE EPI, TR/TE $=4221 / 81 \mathrm{~ms}, b=0-1000 \mathrm{~s} / \mathrm{mm}^{2}$, voxel $=$ 1.8/2.99/5 mm with no gap) with ADC (FLAIR TSE, TR/TE/TI = $6000 / 120 / 2000 \mathrm{~ms}$, voxel $=0.9 / 1.15 / 5 \mathrm{~mm}$ with gap $1 \mathrm{~mm}), \mathrm{T} 2 \mathrm{WI}$ imaging $(\mathrm{TSE}, \mathrm{TSE}$ factor $=23, \mathrm{TR} / \mathrm{TE}=5600 / 110 \mathrm{~ms}$, voxel $=$ $0.9 / 1.14 / 5 \mathrm{~mm}$ ), T2 ${ }^{*} \mathrm{WI}$ imaging $(\mathrm{FFE}, \mathrm{TR} / \mathrm{TE}=653 / 23 \mathrm{~ms}$, flip angle $=18^{\circ}$, voxel $\left.=1.8 / 1.8 / 5 \mathrm{~mm}\right), 3 \mathrm{D}$ time-of-flight $(\mathrm{FFE} \mathrm{T} 1$ inflow, $\mathrm{TR} / \mathrm{TE}=23 / 2.5 \mathrm{~ms}$, voxel $=0.94 / 1.05 / 1.4 \mathrm{~mm}$ ) with maximumintensity-projection reconstructions, first-pass gadolinium PWI (3D gradient echo EPI, Preston, Philips Healthcare, TR/TE $=17 / 25 \mathrm{~ms}$, voxel $=3.59 / 4.51 / 5 \mathrm{~mm}$ ), and pre- and postgadolinium T1WI imaging $(\mathrm{SE}, \mathrm{TR} / \mathrm{TE}=582 / 15$, voxel $=0.94 / 0.94 / 5 \mathrm{~mm})$.
Pretreatment MR imaging and follow up CT scans as well as any additional cerebral imaging performed before patient discharge were reviewed by a stroke neurologist (N.G.) and a neuroradiologist (C.S.) independently, blinded to clinical data. RSCI on MR imaging was defined when fulfilling the following criteria: 1) a parenchymal area markedly hyperintense on FLAIR relative to the surrounding tissue and different from the hyperacute infarct, and 2) mildly-to-markedly hyperintense on DWI with high $b=1000$ relative to surrounding tissue, or 3 ) enhanced on postgadolinium T1WI. Three RSCI patterns on MR imaging were thus distinguishable, as illustrated by Fig 1. To distinguish RSCI and hyperacute IS, which may appear slightly hyperintense on FLAIR, we only considered RSCI if it was clearly separable from the acute infarct area and more hyperintense on FLAIR, reflecting an older lesion. ${ }^{14}$ Small chronic infarcts were distinguished from RSCI and identified as hypointense lesions with a surrounding hyperintense margin on FLAIR, ${ }^{15}$ neither enhanced nor appearing brightly hyperintense on DWI. There were no discrepant cases between the 2 readers. ADC maps were reviewed but were not included as a criterion of the RSCI definition, being either decreased in the RSCI within a few days after ischemic injury or normal in RSCI within a few weeks after ischemia, thus providing redundant data compared with DWI $b=1000$ analysis.

We defined an HT as a hemorrhage occurring within the area of the hyperacute infarct or within an RSCI on any follow-up CT or MR imaging scan before patient discharge. HT was classified according to ECASS imaging criteria as HI-1, HI-2, PH-1, and PH-2. ${ }^{4}$ We defined an EIH as a hemorrhage occurring in a region remote from the visible infarct on pretreatment MR imaging. We defined an $\mathrm{SICH}$, according to ECASS II criteria, as any neurologic deterioration defined as $\geq 4$ points worsening of the NIHSS score or death, associated with HT or $\mathrm{EIH}$ on imaging during inpatient follow-up.

Stroke etiology was assigned according to TOAST criteria ${ }^{16}: \mathrm{Pa}-$ 
MR Imaging Characteristics of Qualifying Lesions (RSCl)

\begin{tabular}{|c|c|c|c|c|c|c|c|c|c|c|c|c|}
\hline No. & $\begin{array}{l}\text { Age } \\
\text { (yr) }\end{array}$ & $\begin{array}{l}\text { Thromb. } \\
\text { IA/IV }\end{array}$ & $\begin{array}{l}\text { Stroke } \\
\text { Etiology }\end{array}$ & $\begin{array}{l}\text { Infarct } \\
\text { Territory }\end{array}$ & $\begin{array}{c}\text { Occluded } \\
\text { Vessel }\end{array}$ & $H T^{b}$ & Pattern Type & $A D C$ & N RSCl & Нуро-GRE & $\begin{array}{l}\text { Max. Diam. } \\
\quad(\mathrm{mm})\end{array}$ & Location $^{c}$ \\
\hline 1 & 63 & IV & Undetermined & R Carot. & M2 & $\mathrm{HI}-1$ & Gad +/DWI- & $\mathrm{N}$ & 1 & No & 41 & Cortical/same arterial territory \\
\hline 2 & 63 & IA & LV Dis. & R Carot. & M1 & - & $\mathrm{Gad}-/ \mathrm{DWI}+$ & D & 1 & No & 5 & Cortical/same arterial territory \\
\hline 3 & 77 & IA & Other & R Carot. & IC & $\mathrm{PH}-2$ & Gad-/DWI+ & $\mathrm{D}$ & 2 & No & 5 & Cortical/same arterial territory \\
\hline 4 & 42 & $\mathrm{IA}$ & Other & R Carot. & M1 & PH-1 & $\mathrm{Gad}+/ \mathrm{DWI}+$ & D & $\Delta$ & No & 15 & Multiple territories \\
\hline 5 & 63 & IV & LV Dis. & L Carot. & $\mathrm{CC}+\mathrm{M} 1$ & $\mathrm{HI}-2$ & $\mathrm{Gad}-/ \mathrm{DWI}+$ & $\mathrm{D}$ & 1 & $\mathrm{~N}$ & 7 & Cortical/same arterial territory \\
\hline 6 & 74 & IV+MT & LV Dis. & R Carot. & $\mathrm{CC}+\mathrm{M} 1$ & PH-1 & $\mathrm{Gad}+/ \mathrm{DWI}+$ & $\mathrm{D}$ & 3 & $\mathrm{~N}$ & 15 & Cortical/same arterial territory \\
\hline 7 & 49 & IV & Undetermined & L Carot. & $\mathrm{CC}+\mathrm{M} 1$ & - & $\mathrm{Gad}+/ \mathrm{DWI}-$ & $\mathrm{N}$ & 1 & No & 11 & Cortical/same arterial territory \\
\hline 8 & 36 & IA & Other & BA & $\mathrm{BA}$ & - & $\mathrm{Gad}-/ \mathrm{DWI}+$ & D & 1 & No & 23 & Cortical/same arterial territory \\
\hline 9 & 40 & IA & Cardioembolic & L Carot. & M1 & - & $\mathrm{Gad}+/ \mathrm{DWI}-$ & N & 2 & No & 13 & Cortical/same arterial territory \\
\hline 10 & 78 & IV & LV Dis. & L Carot. & $\mathrm{CC}+\mathrm{M} 1$ & $\mathrm{HI}-2$ & $\mathrm{Gad}+/ \mathrm{DWI}-$ & $\mathrm{N}$ & 1 & Yes & 15 & Cortical/other arterial territory \\
\hline
\end{tabular}

Note:-IA indicates intra-arterial; Thromb., thrombolysis; HT, hemorrhagic transformation; N, normal ADC compared with the contralateral side; D, decreased ADC compared with contralateral side; N RSCI, number of areas of RSCI per patient; hypo-GRE, hypointensity on T2 gradient-echo-T2WI within the RSCl area; Max. Diam., maximum diameter of RSCI in millimeters measured on axial FLAIR; LV Dis., large-vessel disease etiology; Other, other etiology; R Carot., right internal carotid artery; L Carot., left internal carotid artery; M2, second segment of the middle cerebral artery; M1, first segment of the middle cerebral artery; IC, intracranial internal carotid artery; CC, cervical internal carotid artery; BA, basilar artery; MT, mechanical thrombectomy; Gad+, gadolinium enhancement on T1Wl; Gad-, lack of gadolium enhancement on T1Wl.

according to the TOAST classification, patients younger than 56 years with associated patent foramen ovale and atrial septum aneurysm (patient 9) were classified under "cardioembolic."

"Other" etiology was myeloproliferative-disorder-associated coagulopathy in patient 3; paraneaoplastic coagulopathy in patient 4; and vertebral artery dissection in patient 8.

${ }^{b}$ According to the ECASS definition. ${ }^{4}$

${ }^{c}$ Location of the RSCl was compared with the acute infarct arterial territory.

tients with middle cerebral artery occlusion and definite ipsilateral carotid atherosclerotic stenosis or occlusion ("tandem occlusion") only were assigned to large-vessel disease. Basilar artery occlusion was, as well, considered as large-vessel disease when atherosclerotic stenosis was identified on cervical or intracranial vertebral or basilar arteries without another source of embolism.

\section{Statistical Analysis}

Data were expressed either as frequency and percentages or as a median and IQR. Comparative analysis was performed by using the 2-sided Fisher exact test or Wilcoxon-Mann-Whitney test. J.P.B. performed statistical analysis with SAS, Version 8.1 (SAS Institute, Cary, North Carolina) with the $\alpha$ level set at 5\%.

\section{Results}

\section{Patient Characteristics}

The baseline characteristics of the 86 patients studied are shown in On-Line Table 1: Median age was 66 years (range, 61-78 years), median NIHSS score on admission was 15 (range, 9-19), and median symptom-to-needle time was 3.1 hours (range, $2.8-4.5$ hours). rtPA administration was either intravenous or intra-arterial in 66\% (57/86) and 34\% (29/86) of patients, respectively.

\section{Incidence and Characteristics of RSCI}

We identified 10 of 86 patients (12\%) with at least 1 RSCI on pretreatment MR imaging, which resulted in a total number of 17 RSCI areas. On-line Table 2 shows the main comparative baseline and acute stroke characteristics of patients with and without RSCI. All demographic data and main vascular risk factors, history of stroke or TIA in the previous 3 months, and clinical and biologic admission risk factors for HT were similar. When we considered stroke etiology by using the TOAST classification, ${ }^{16}$ the 2 groups appeared significantly different $(P<.001)$ : Large-vessel disease was more frequent $(40 \%$ versus $5 \%)$ in patients with RSCI. Clinical and imaging acute stroke characteristics on admission were comparable except for the occlusion of a major intracranial artery (internal ca- rotid or basilar artery) on MRA, which was twice as frequent in patients with RSCI, though not significant (60\% versus $28 \%$, $P=.06)$.

Table 1 summarizes the characteristics of patients with RSCI. All except 1 (9/10) had an acute infarct in the anterior circulation territory related to the occlusion of an intracranial internal carotid artery $(n=1)$ or middle cerebral artery $(n=$ 4 ) or tandem occlusion of both cervical internal carotid and middle cerebral arteries $(n=4)$. Four patients $(40 \%)$ had multiple RSCIs. RSCIs were distributed, in most cases (80\%), within the acute infarct arterial territory, mostly in cortical zones bordering the middle cerebral artery territory. One patient had multiple RSCIs in both posterior and anterior circulation territories. This patient had an RSCI located in the right anterior circulation territory, while acute infarct had occurred in the left anterior territory. RSCI size ranged from 5 to $41 \mathrm{~mm}$ (mean value, $15 \mathrm{~mm}$ ) on axial FLAIR images with the maximal diameter measured. One RSCI (patient 10 in On-line Table 1) was characterized on pretreatment MR imaging by a "Gad +/ DWI-" pattern and hemosiderin staining on T2*WI, which indicated a subacute silent hemorrhagic infarction with still ongoing BBBD. As shown in On-line Table 1, all RSCI hyperintense on DWI showed decreased ADC compared with the contralateral side, whereas RSCIs not hyperintense on DWI had normal ADCs.

Among the 86 patients, 34 (40\%) had an HT within the acute infarct during inpatient follow-up, with no statistically significant difference between patients with $(n=6 / 10 ; 60 \%)$ or without RSCI $(n=28 / 76,37 \%)$. The SICH rate was also similar in both groups (Table 2). Additional cerebral imaging after the day 1 CT scan was performed in $80 \%$ of patients with RSCI and $81 \%$ of patients without RSCI (Table 2). Follow-up imaging was a CT scan in $71 \%$ of cases, without any statistical differences between groups. HT was visible on follow-up MR imaging only as a hypointensity on $\mathrm{T} 2{ }^{*} \mathrm{WI}$, but not on follow-up CT scans, in 2 patients, 1 in each group.

No hemorrhagic transformation was seen within any RSCI lesion on either postthrombolysis CT scans on day 1 or any 


\begin{tabular}{|c|c|c|c|}
\hline \multicolumn{4}{|c|}{$\begin{array}{l}\text { Table 2: Comparison of postthrombolysis HT incidence between } \\
\text { groups }\end{array}$} \\
\hline HT & $\begin{array}{c}\text { Group without RSCI } \\
(n=76) \\
(\text { No.) }(\%)\end{array}$ & $\begin{array}{c}\text { Group with RSCI } \\
(n=10) \\
(\text { No.) }(\%)\end{array}$ & $P$ \\
\hline $\begin{array}{c}\text { In-hospital additional } \\
\text { cerebral imaging }\end{array}$ & $61(81)$ & $8(80)$ & 1.0 \\
\hline HT & $28(37)$ & $6(60)$ & .1 \\
\hline $\mathrm{EIH}$ & $1(1)$ & 0 & 1.0 \\
\hline $\mathrm{SICH}$ & $6(8)$ & $1(10)$ & 1.0 \\
\hline
\end{tabular}

further imaging when available. An EIH, which was also an SICH, occurred in 1 patient (1\%) without any RSCI (On-Line Fig 1) and without any evidence of structural abnormality on the pretreatment MR imaging within the area of the EIH.

\section{Discussion}

We report here a systematic evaluation of RSCI incidence in prethrombolysis MR imaging in acute IS. RSCIs were not rare, because $12 \%$ of patients had at least 1 RSCI, with multiple RSCIs seen in $40 \%$ of patients. RSCIs were of small size and most often distributed in cortical zones bordering the arterial territory of the occluded intracranial artery on MRA. This distribution could indicate that microembolic thrombi had occurred in the hours to weeks prior, just as TIAs ${ }^{17,18}$ or recurrent silent ischemic lesions ${ }^{19}$ can occur in the hours to days before IS. Moreover, we found that the presence of RSCI was associated, on univariate analysis, with large-vessel disease and with the presence of large-vessel occlusion (basilar or carotid artery), though not significantly. This result is in agreement with the study of Kang et al, ${ }^{19}$ who found that patients with silent ischemic lesion recurrence on follow-up MR imaging after IS had more frequent large-artery atherosclerosis than patients without silent lesion recurrence. One explanation might be that RSCIs, which occurred from hours to weeks before acute IS onset, could be, in some cases, asymptomatic embolic markers of atherosclerotic occlusion or stenosis of a large artery, leading finally to IS, as suggested previously. ${ }^{19}$

Multimodal MR imaging is highly sensitive in detecting both small acute or subacute infarcts ${ }^{7,12,20}$ and early or delayed BBBD within the infarct area. ${ }^{8-11}$ An acute infarct appears hyperintense on FLAIR and DWI with a high b-value within the first few hours postonset; then, during the subacute phase, hyperintensity increases for approximately 10 days before decreasing gradually on DWI during a period of $\leq 2$ months. ${ }^{14}$ Also, contrast enhancement on postgadolinium T1WI occurs very early after IS, within the first hours to days, and persists for several weeks to months thereafter..$^{8-11,21}$ The association of a marked hyperintensity on both FLAIR and DWI or the association of a marked hyperintensity on FLAIR with contrast enhancement on postgadolinium T1WI thus allowed us to define subacute ischemic areas on pretreatment MR imaging, which dated from a few days to several weeks before acute stroke onset.

The principal outcome is that none of the RSCI areas evolved to HT postthrombolysis with rtPA. Several predictive parameters of postthrombolysis SICH have been identified. ${ }^{3,4,22,23}$ Most intriguing, approximately $20 \%$ of SICHs after intravenous rtPA in patients with stroke occurred in regions without visible ischemic changes on pretreatment CT scans; they were EIHs. ${ }^{4,5}$ One study hypothesized that RSCI could account for cerebral hemorrhages that occur remote from the acute infarct. Indeed, several MR imaging studies have shown that early BBBD in acute stroke, whether after thrombolysis or not, was associated with HT or $\mathrm{SICH},{ }^{8,10,11,24,25}$ though HT occurred in acutely injured tissue rather than in regions distant from the acute infarct field, which may reflect early reperfusion-related injury to the ischemic microvasculature. ${ }^{4,10,14}$

Sloan et $\mathrm{al}^{26}$ retrospectively reviewed 25 patients from an acute myocardial infarction trial who had a SICH after rtPA and heparin therapy. Among those 25 patients, neuropathologic findings were available in 5 patients. It was demonstrated that SICH was an HT of a "clinically silent" periventricular cerebral infarct in 1 patient. HT of a silent cerebral infarct after thrombolysis thus seems to be plausible in patients with stroke as well. Our results indicate, on the contrary, that RSCI on MR imaging, though rather frequent, did not lead to HT and did not account for the only case of EIH that occurred. This finding is of great clinical relevance for daily acute stroke management because stroke neurologists may currently exclude a significant proportion of eligible patients with acute stroke from routine thrombolysis after discovering RSCI on pretreatment MR imaging, which could be considered as a "missed chance" on the basis of our results.

Other potential mechanisms leading to EIH after rtPA may be cerebral amyloid angiopathy, ${ }^{26}$ angiopathy with microbleeds, ${ }^{27}$ arteriovenous malformations, vasculitis, coagulopathies, and post-rtPA-related hypofibrinogenemia. ${ }^{4,26}$ Nevertheless, EIH remains unpredictable in patients without any identified structural abnormalities on pretreatment MR imaging within the area of postthrombolysis $\mathrm{EIH}^{28}$; thus, the mechanisms that operate in the minority of cases are still open to speculation.

This study has some limitations, because the analysis was retrospective and small HTs within RSCI areas could have been missed because repeated imaging controls were not systematically implemented. However, all patients had CT scans if neurologic deterioration occurred, making an underestimate of SICH very unlikely. In our cohort, approximately onethird of patients received intra-arterial rtPA, which thus might have lowered the risk of EIH, but no published data have confirmed this hypothesis to date, to our knowledge. Last, a RSCI of a larger size might have been considered by the stroke neurologist as a contraindication for rtPA administration, thus excluding such patients from the study. EIH occurred rarely, in only $1.3 \%$ of patients treated with rtPA of the cohort or rtPA trials ${ }^{5}$; this low incidence makes the study of potential mechanisms challenging, except by a multicenter analysis including hundreds of patients with prethrombolysis MR imaging scans. Therefore, it is not possible to completely refute the role of prior subacute infarcts in HT occurring outside the ischemic area. A prospective study analyzing the locations of EIH in a cohort of rtPA-treated patients with RSCI could provide more insight into this issue.

\section{Conclusions}

These preliminary results require validation but suggest that small RSCIs are rather frequent, might not pose a higher risk of 
postthrombolysis HT, and may not account for some cases of EIH.

\section{References}

1. Furlan A, Higashida R, Wechsler L, et al. Intra-arterial prourokinase for acute ischemic stroke: the PROACT II study-a randomized controlled trial. Prolyse in Acute Cerebral Thromboembolism. JAMA 1999;282:2003-11

2. Hacke W, Donnan G, Fieschi C, et al, for the ATLANTIS, ECASS, and NINDS rtPA Study Group Investigators. Association of outcome with early stroke treatment: pooled analysis of ATLANTIS, ECASS and NINDS rtPA stroke trials. Lancet 2004;363:768-74

3. Khatri P, Wechsler LR, Broderick JP. Intracranial hemorrhage associated with revascularization therapies. Stroke 2007;38:431-40

4. Trouillas P, von Kummer R. Classification and pathogenesis of cerebral hemorrhages after thrombolysis in ischemic stroke. Stroke 2006;37:556-61

5. Intracerebral hemorrhage after intravenous t-PA for ischemic stroke: the NINDS t-PA Stroke Study Group. Stroke 1997;28:2109-18

6. De Keyser J, Gdovinová Z, Uyttenboogaart M, et al. Intravenous alteplase for stroke: beyond the guidelines and in particular clinical situations. Stroke 2007; 38:2612-18. Epub 2007 Jul 26

7. Albers GW, Lansberg MG, Norbash AM, et al. Yield of diffusion-weighted MRI for detection of potentially relevant findings in stroke patients. Neurology 2000;54:1562-67

8. Kim EY, Na DG, Kim SS, et al. Prediction of hemorrhagic transformation in acute ischemic stroke: role of diffusion weighted imaging and early parenchymal enhancement. AJNR Am J Neuroradiol 2005;26:1050-55

9. Karonen JO, Partanen PL, Vanninen RL, et al. Evolution of MR contrast enhancement patterns during the first week after acute ischemic stroke. AJNR Am J Neuroradiol 2001;22:103-11

10. Latour LL, Kang DW, Ezzeddine MA, et al. Early blood-brain barrier disruption in human focal brain ischemia. Ann Neurol 2004;56:468-77

11. Kastrup A, Gröschel K, Ringer TM, et al. Early disruption of the blood-brain barrier after thrombolytic therapy predicts hemorrhage in patients with acute stroke. Stroke 2008;39:2385-87

12. Chalela JA, Kidwell CS, Nentwich LM, et al. Magnetic resonance imaging and computed tomography in emergency assessment of patients with suspected acute stroke: a prospective comparison. Lancet 2007;369:293-98

13. Schellinger PD, Thomalla G, Fiehler J, et al. MRI-based and CT-based thrombolytic therapy in acute stroke within and beyond established time windows: an analysis of 1210 patients. Stroke 2007;38:2640-45

14. Lansberg MG, Thijs VN, O'Brien MW, et al. Evolution of apparent diffusion coefficient, diffusion-weighted, and T2-weighted signal intensity of acute stroke. AJNR Am J Neuroradiol 2001;22:637-44

15. Kimberly WT, Gilson A, Rost NS, et al. Silent ischemic infarcts are associated with hemorrhage burden in cerebral amyloid angiopathy. Neurology 2009;72:1230-35

16. Adams HP Jr, Bendixen BH, Kappelle LJ, et al. Classification of subtype of acute ischemic stroke: definitions for use in a multicenter clinical trial-TOAST. Trial of Org 10172 in Acute Stroke Treatment. Stroke 1993;24:35-41

17. Purroy F, Montaner J, Rovira A, et al. Higher risk of further vascular events among transient ischemic attack patients with diffusion-weighted imaging acute ischemic lesions. Stroke 2004;35:2313-19

18. Coutts SB, Simon JE, Eliasziw M, et al. Triaging transient ischemic attack and minor stroke patients using acute magnetic resonance imaging. Ann Neurol 2005;57:848-54

19. Kang DW, Lattimore SU, Latour LL, et al. Silent ischemic lesion recurrence on magnetic resonance imaging predicts subsequent clinical vascular events. Arch Neurol 2006;63:1730-33

20. Warach S, Gaa J, Siewert B, et al. Acute human stroke studied by whole brain echo planar diffusion-weighted magnetic resonance imaging. Ann Neurol 1995;37:231-41

21. Imakita S, Nishimura T, Yamada N, et al. Magnetic resonance imaging of cerebral infarction: time course of Gd-DTPA enhancement and CT comparison. Neuroradiology 1988;30:372-78

22. Tanne D, Kasner SE, Demchuk AM, et al. Markers of increased risk of intracerebral hemorrhage after intravenous recombinant tissue plasminogen activator therapy for acute ischemic stroke in clinical practice: the Multicenter rtPA Stroke Survey. Circulation 2002;105:1679-85

23. Derex L, Hermier M, Adeleine $\mathrm{P}$, et al. Clinical and imaging predictors of intracerebral haemorrhage in stroke patients treated with intravenous tissue plasminogen activator. J Neurol Neurosurg Psychiatry 2005;76:70-75

24. Thomalla G, Sobesky J, Köhrmann M, et al. Two tales: hemorrhagic transformation but not parenchymal hemorrhage after thrombolysis is related to severity and duration of ischemia-MRI study of acute stroke patients treated with intravenous tissue plasminogen activator within 6 hours. Stroke 2007;38:313-18

25. Vo KD, Santiago F, Lin W, et al. MR imaging enhancement patterns as predictors of hemorrhagic transformation in acute ischemic stroke. AJNR Am JNeuroradiol 2003;24:674-79

26. Sloan MA, Price TR, Petito CK, et al. Clinical features and pathogenesis of intracerebral hemorrhage after rtPA and heparin therapy for acute myocardial infarction: the Thrombolysis in Myocardial Infarction (TIMI) II pilot and randomized clinical combined experience. Neurology 1995;45:649-58

27. Kidwell CS, Saver JL, Villablanca JP, et al. Magnetic resonance imaging detection of microbleeds before thrombolysis: an emerging application. Stroke 2002;33:95-98

28. Hill MD, Barber PA, Demchuk AM, et al. Symptomatic hemorrhage after alteplase therapy not due to silent ischemia. BMC Neurol 2001;18:1:1 